\title{
Prevalência de cuidados paliativos em pacientes com síndrome de imobilidade em um serviço de atenção domiciliar
}

\author{
Prevalence of palliative care in patients with immobility \\ syndrome in a home care attention service
}

\author{
Rafaela Espindola ${ }^{1}$, Clovis Cechinel ${ }^{2}$, Ricardo Papp Moretti ${ }^{3}$
}

Resumo

\begin{abstract}
Introdução: a síndrome de imobilidade (SI) é definida por critérios clínicos específicos, a saber, presença dos dois critérios maiores, declínio cognitivo e múltiplas contraturas e pelo menos dois dos menores, disfagia, afasia, lesão por pressão e dupla incontinência, tendo indicação de cuidados paliativos desde o momento de sua identificação. Objetivo: determinar a prevalência da SI em pacientes em atendimento domiciliar e verificar a inclusão destes em cuidados paliativos. Métodos: estudo documental, descritivo-exploratório e retrospectivo, coletados do prontuário dos pacientes do Serviço de Atendimento Domiciliar de Curitiba, no período de 1 a 31 de julho de 2018, totalizando 566 pacientes. Critérios de inclusão: idade igual ou superior a 18 anos, estar em atendimento domiciliar, apresentar critérios para SI. Critérios de exclusão: dados incompletos que impeçam a caracterização da SI. Foi utilizado o teste de associação de qui-quadrado e o teste da ANOVA. Considerou-se o $p \leq 0,05$ com significância estatística. Resultados: 56 (9,9\%) apresentaram critérios para SI. A ocorrência da SI no sexo feminino foi de $13,1 \%$ e no masculino $6,1 \%$, com significância estatística $(p=0,0218)$. A média de idade de 72,43 . Quanto aos critérios menores, $n=56(100 \%)$ tinham dupla incontinência, $n=48(83,9 \%)$ disfagia, $n=41(73,2 \%)$ afasia e $\mathrm{n}=31(55,4 \%)$ lesões por pressão. Dos pacientes com SI, $44,46 \%$ estavam em cuidados paliativos. Conclusão: a SI foi associada ao sexo feminino, a média de idade foi 72,43 anos. A prevalência foi de $9,9 \%$, dos quais somente $44,46 \%$ estavam em cuidados paliativos, o que nos aponta que o atendimento domiciliar não está ofertando aos seus pacientes o melhor atendimento possível.
\end{abstract}

Palavras-chave: Imobilização. Cuidados paliativos. Serviços de assistência domiciliar.

\footnotetext{
${ }^{1}$ Residência em Geriatria pelo Hospital do Idoso Zilda Arns, Curitiba, Paraná, Brasil. Fundação Estatal de Atenção Especializada em Saúde de Curitiba; Serviço de Atendimento Domiciliar Programa Melhor em Casa Curitiba, Curitiba, Paraná, Brasil.

${ }^{2}$ Mestrado em Enfermagem pela Universidade Federal do Paraná (UFPR), Curitiba, Paraná, Brasil. Coordenador Médico do Programa Melhor em Casa Hospital do Idoso Zilda Arns, Curitiba, Paraná, Brasil. E-mail: cechinelc@hotmail.com

${ }^{3}$ Médico pela Pontifícia Universidade Católica do Paraná(PUC-PR), Curitiba, Paraná, Brasil. Médico do Serviço de Atendimento Domiciliar Programa Melhor em Casa Curitiba, Curitiba, Paraná, Brasil.
} 


\begin{abstract}
Introduction: the immobility syndrome (IS) is defined by specific clinical criteria, namely, presence of the two major criteria, cognitive decline and multiple contractures and at least two of the smallest, dysphagia, aphasia, pressure injury and double incontinence and indicates palliative care from the moment of its identification. Objective: to determine the prevalence of IS in patients in home care and to verify their inclusion in palliative care. Methods: this is a documentary, descriptiveexploratory and retrospective study, collected from patients' records of the Home Care Service of Curitiba, from 1 to 31 July 2018, totaling 566 patients. Inclusion criteria: age equal to or greater than 18 years, being in home care, presenting criteria for IS. Exclusion criteria: incomplete data that prevent the characterization of the IS. The chi-square association test and the ANOVA test were used. The $\mathrm{p} \leq 0.05$ was considered statistically significant. Results: $\mathrm{n}=56(9.9 \%)$ presented criteria for IS. The occurrence of IS in the female sex was $13.1 \%$ and in the male, $6.1 \%$, with statistical significance $(\mathrm{p}=0.0218)$. The mean age was 72.43 . Regarding the minor criteria $n=56(100 \%)$ had double incontinence, $n=48(83.9 \%)$ dysphagia, $n=41(73.2 \%)$ aphasia, and $n=31(55.4 \%)$ pressure injuries. Of the patients with IS $44.46 \%$ were in palliative care. Conclusion: IS was associated with females, mean age was 72.43 years. The prevalence was $9.9 \%$, of which only $44.46 \%$ were in palliative care, which indicates that home care is not offering its patients the best care possible.
\end{abstract}

Keywords: Immobilization. Palliative care. Home care services.

\section{Introdução}

A síndrome de imobilidade (SI) é conhecida entre os geriatras, porém pouco conhecida entre as demais especialidades médicas, ${ }^{(1)}$ acomete indivíduos com enfermidades incapacitantes, o que culmina na supressão dos movimentos articulares. As causas do comprometimento da mobilidade são multifatoriais, predominando as neurológicas e musculoesqueléticas. Independente da condição que a desencadeou, ela pode evoluir com complicações: redução de massa muscular e óssea, acúmulo de secreção pulmonar, infecções, alterações metabólicas, obstipação intestinal, desnutrição, lesão por pressão, depressão e isolamento social. ${ }^{(2)}$

A SI é uma entidade ainda não definida com clareza na literatura internacional, a qual não utiliza essa terminologia, não sendo possível avaliar prevalência da SI em trabalhos internacionais. $\mathrm{Na}$ literatura nacional constatou-se apenas um estudo de prevalência da SI, conduzido em uma enfermaria de geriatria, que evidenciou 25,53\% dos pacientes com síndrome. ${ }^{(3)}$

Para se chegar ao diagnóstico é necessário que o paciente tenha dois critérios maiores e pelo menos dois critérios menores. Os critérios maiores consistem em déficit cognitivo médio a grave e múltiplas contraturas articulares. Já os critérios menores são sinais de sofrimento cutâneo ou lesão por pressão, disfagia leve a grave, dupla

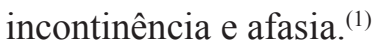

As próprias características diagnósticas da SI já sinalizam a impossibilidade de locomoção, o que evidencia a modalidade de atendimento domiciliar como um importante ponto da Rede de Atenção à Saúde (RAS) no cuidado destes pacientes. ${ }^{(4)}$ A Atenção Domiciliar (AD) não tem caráter substitutivo, mas sim complementar aos demais serviços e integra um conjunto de ações de saúde prestadas em domicílio como prevenção e tratamento de doenças, reabilitação, promoção à saúde e cuidados paliativos, o que garante a continuidade de cuidados. ${ }^{(5)}$

A SI tem indicação incontestável de cuidados paliativos, sendo a linha de cuidado que irá trazer maior qualidade de vida ao paciente, seus familiares e cuidadores. Cuidados paliativos consistem no cuidado holístico dos indivíduos com importante sofrimento relacionado com a presença de uma doença grave. ${ }^{(6)}$

Frente à complexidade do cuidado da SI, é imperioso perceber estes pacientes em cuidados 
paliativos. Devido a isso, este estudo objetivou determinar a prevalência da SI e da inclusão de cuidados paliativos nos pacientes acompanhados pelo Serviço de Atenção Domiciliar (SAD).

\section{Métodos}

Estudo transversal quantitativo de caráter retrospectivo no período amostral de 1 a 31 de julho de 2018, utilizando dados secundários de planilhas gerenciais e prontuário de pacientes acompanhados em um SAD de Curitiba. O número total de pacientes atendidos foi de 584 , sendo que 18 foram excluídos por terem idade inferior a 18 anos, totalizando 566 para análise.

O SAD objetiva reduzir a demanda por atendimento hospitalar ou redução do período de permanência de usuários internados; preza pela humanização da atenção à saúde, com a ampliação da autonomia dos usuários, além da otimização dos recursos financeiros e estruturais. Organizase por meio de 10 Equipes Multiprofissionais de Atenção Domiciliar (EMAD) e três Equipes Multiprofissionais de Apoio (EMAP), atuando complementarmente aos cuidados da Atenção Primária, serviços de urgência e emergência e substitutivo ou suplementar à internação hospitalar.

A coleta ocorreu em setembro de 2018, mediante dados do Sistema de Gestão Hospitalar Philips Tazy ${ }^{\circledR}$, tabulados em planilhas eletrônicas do Microsoft Excel ${ }^{\circledR}$, e incluía dados sociodemográficos, clínicos, causas do imobilismo, além da obtenção de informações para caracterização da síndrome. Os critérios diagnósticos para síndrome de imobilidade são: presença dos dois critérios maiores - declínio cognitivo e múltiplas contraturas - e pelo menos dois dos quatro menores: lesão por pressão, disfagia leve a grave, dupla incontinência e afasia.

Critérios de inclusão: idade igual ou superior a 18 anos, estar em atendimento domiciliar, apresentar critérios para SI descritos na evolução de prontuário. Subsequente estabeleceram-se os critérios de exclusão: dados incompletos que impeçam a caracterização da SI.
Para avaliar se a síndrome estava associada com o sexo foi utilizado o teste de associação de qui-quadrado, e para avaliar a relação com a idade foi utilizado o teste da ANOVA. A aplicação do teste da ANOVA depende da homogeneidade de variâncias entre os grupos, que foi avaliada através do teste de Levene, e a normalidade dos resíduos, que foi avaliada via teste de Shapiro Wilk. Quando tais exigências não foram atendidas foi utilizado o teste de Kruskal - Wallis, que é o teste não paramétrico correspondente ao ANOVA (ao invés de testar se a média da variável é a mesma, ele avalia a distribuição (postos) da variável, e é livre de distribuição). Considerou-se o $p \leq 0,05$ com significância estatística. O software utilizado para desenvolver a análise foi o R (R Core Team, 2018).

O estudo recebeu parecer favorável 3.031.860 do Comitê de Ética em Pesquisa em Seres Humanos do Setor de Ciências da Saúde, sendo aprovado sob o registro CAAE 97552718.1.0000.0101.

\section{Resultados}

A amostra final foi constituída por 566 pacientes, dentre estes, $56(9,9 \%)$ apresentaram critérios para SI; a média de idade dos pacientes com SI foi de 72,43 anos, com desvio padrão de 19,7 (21 a 97 anos). A ocorrência da síndrome de imobilidade no sexo feminino foi de $13,1 \%$ e no masculino $6,1 \%$, com significância estatística $(p=0,0218)$. Ao correlacionar SI e idade, não houve significância estatística $(p=0,2581)$, concluindo-se que não há diferença na média de idade dos pacientes em relação ao fato de o paciente ter a síndrome.

$\mathrm{Na}$ Tabela 1, descreve-se o perfil sociodemográfico dos pacientes categorizados com síndrome de imobilidade, sendo 69,64\% do sexo feminino, $42,86 \%$ viúvos, $10,71 \%$ com sonda vesical de demora e $26,79 \%$ com sonda nasoenteral. 
Tabela 1 - Perfil sociodemográfico dos pacientes com síndrome de imobilidade. Curitiba-PR, Brasil, 2018.

\begin{tabular}{llcc}
\hline Variável & & $\mathbf{n}$ & $\mathbf{\%}$ \\
\hline GÊNERO & Feminino & 39 & 69,64 \\
ESTADO CIVIL & Masculino & 17 & 30,36 \\
& Viúvo (a) & 24 & 42,86 \\
& Casado (a) & 20 & 35,71 \\
CUIDADOS PALIATIVOS & Solteiro (a) & 9 & 16,07 \\
& Divorciado (a) & 3 & 5,36 \\
& Não & 26 & 46,43 \\
SVD & Sim & 25 & 44,64 \\
& Não (família não aceitou) & 5 & 8,93 \\
SVA & Não & 50 & 89,29 \\
SNE & Sim & 6 & 10,71 \\
& Não & 56 & 100,00 \\
\hline
\end{tabular}

Fonte: Autores

Observa-se na Figura 1 que a principal causa demência vascular (41\%), seguido da demência de da SI foi acidente vascular encefálico - AVE ou Alzheimer (23\%) e trauma de crânio (11\%).

Figura 1 - Gráfico de barras para as causas de SI. Curitiba-PR, Brasil.

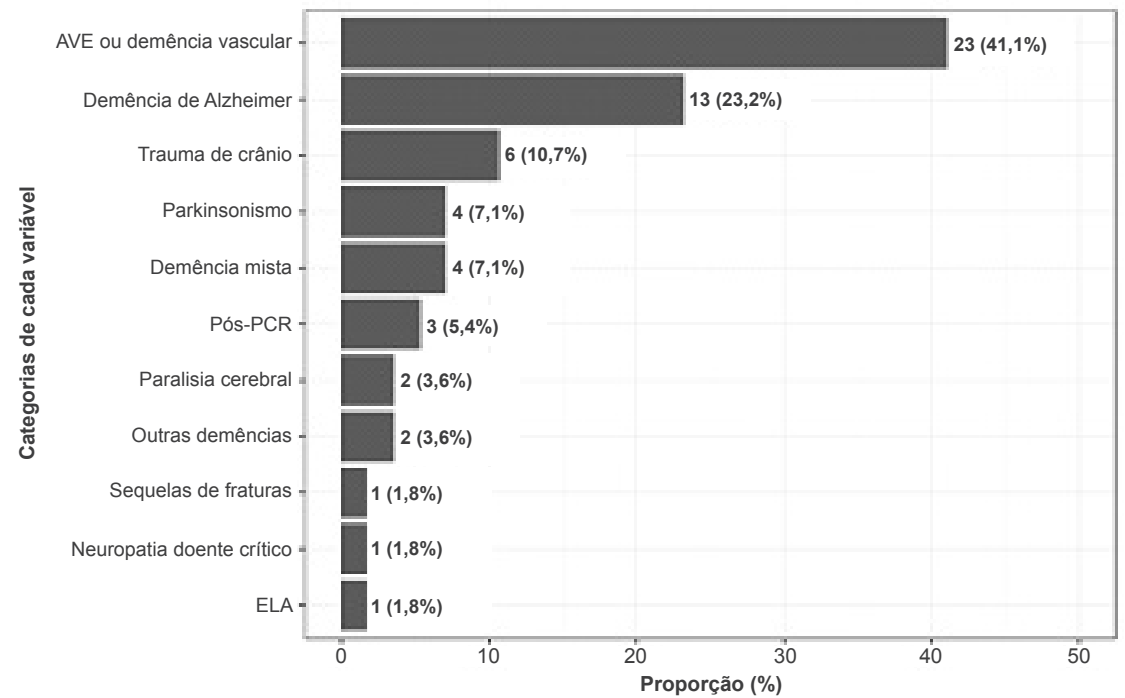

Fonte: Autores 
Quanto aos critérios diagnósticos, os disfagia, $n=41 \quad(73,2 \%)$ afasia e $n=31 \quad(55,4 \%)$ menores apresentaram a seguinte frequência: lesões por pressão (Figura 2). $\mathrm{n}=56(100 \%)$ dupla incontinência, $\mathrm{n}=47(83,9 \%)$

Figura 2 - Gráfico de barras com critérios diagnósticos da SI. Curitiba-PR, Brasil.

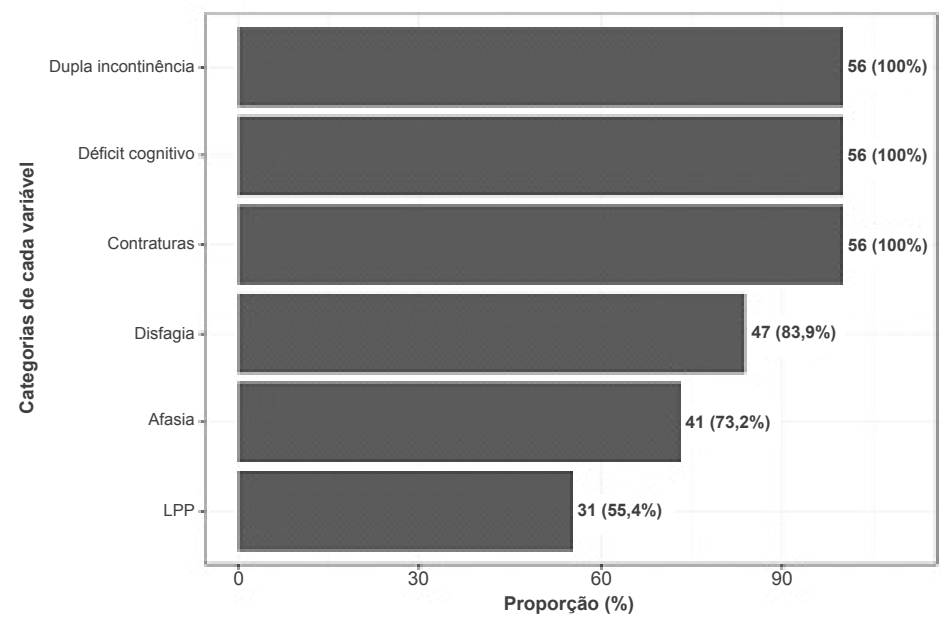

Fonte: Autores

$\mathrm{Na}$ Figura 3, foram colocadas as principais a $48 \%$ dentre os que não apresentaram trauma de causas de síndrome de imobilidade, comparando crânio), enquanto $100 \%$ dos que apresentaram com o restante dos casos. Percebe-se que, dentre demência mista foram submetidos a cuidados os que apresentam trauma de crânio $(n=6), 16,7 \%$ paliativos (comparado a 40,4\% dentre os que não eram submetidos a cuidados paliativos (comparado sofreram demência mista).

Figura 3 - Relação entre cuidados paliativos e as causas de síndrome de imobilidade.

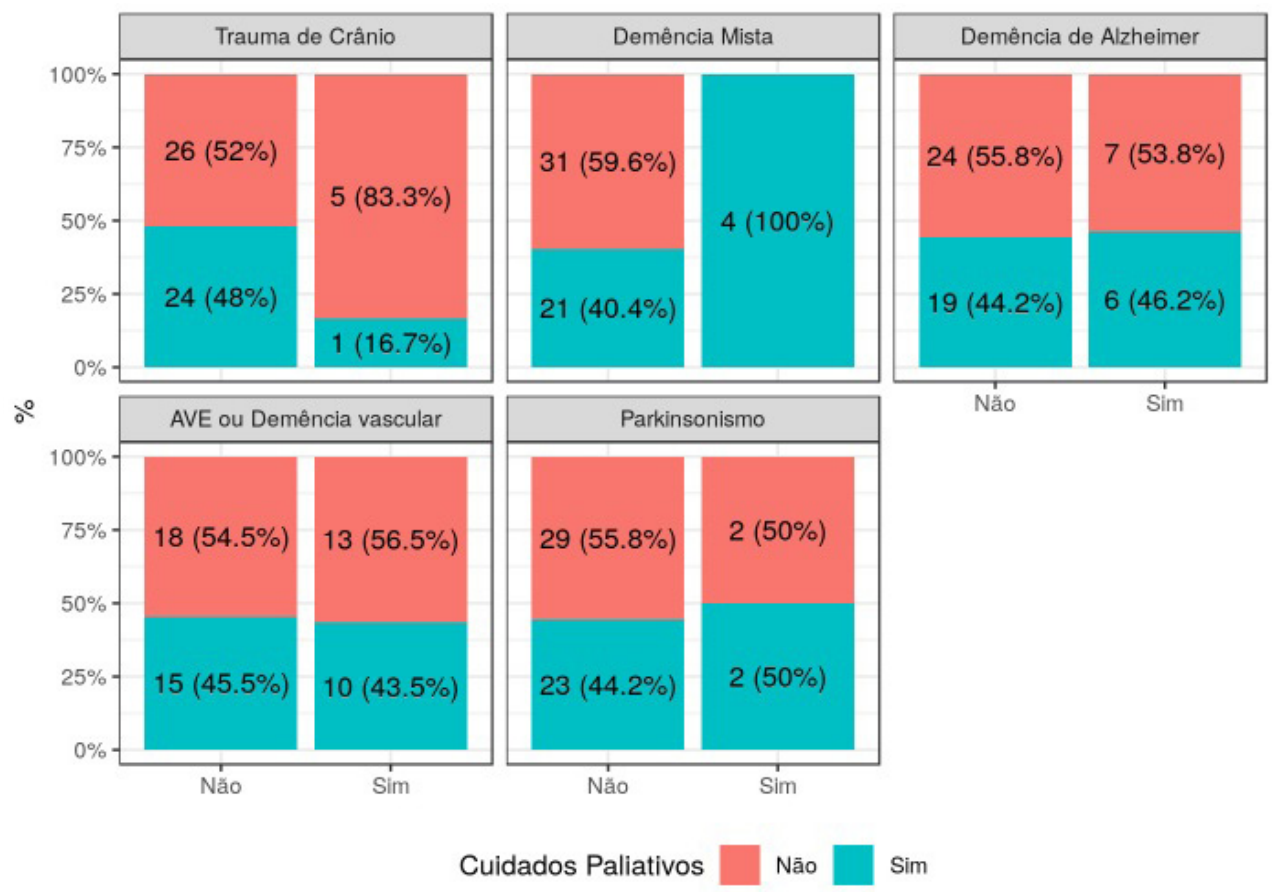

Fonte: Autores 
$\mathrm{Na}$ Tabela 2, correlacionou-se as causas de imobilismo e inclusão em cuidados paliativos; observou-se que apenas a demência mista teve um $p$ significativo: $p=0,0344$.

Tabela 2 - Relação das causas de síndrome de imobilização e cuidados paliativos. Curitiba-PR, Brasil.

\begin{tabular}{lc}
\hline Covariáveis & valor $\boldsymbol{p}$ \\
\hline Demência mista & 0,0344 \\
Trauma de crânio & 0,2097 \\
Demência de Alzheimer & 1 \\
AVE ou demência vascular & 1 \\
Parkinsonismo & 1 \\
\hline
\end{tabular}

Fonte: Autores

*Teste exato de Fisher, $p \leq 0,05$.

\section{Discussão}

A prevalência da SI foi de 9,6\%. Esse valor foi bem inferior ao encontrado em um estudo nacional realizado em uma enfermaria de geriatria que mostrou uma prevalência de 25,53\%. ${ }^{(3)}$ Esta diferença pode ser justificada pela inclusão de maiores de 18 anos neste estudo, e não apenas maiores de 60 anos, e pela metodologia utilizada no estudo.

A principal causa da SI foi acidente vascular encefálico / demência vascular (41\%), seguido da demência de Alzheimer - DA (23\%), trauma de crânio (11\%), parkinsonismo (7\%) e demência mista (7\%). Os critérios da SI sinalizam uma população com baixa funcionalidade, sendo as mesmas causas de um estudo conduzido na Atenção Primária de Londrina-PR com 73 pacientes em cuidados paliativos (CP), no qual as síndromes demenciais e as sequelas por acidente vascular cerebral foram as condições clínicas mais frequentes com $27 \%$ e $26 \%$, respectivamente; seguidas de distúrbios osteoarticulares: $12 \%$, outras doenças neurológicas: $11 \%$, cardiopatias: $10 \%$ e câncer: $8 \% .^{(7)}$
Um estudo realizado em uma instituição de longa permanência para idosos também encontrou predominância de doenças cerebrovasculares e síndromes demenciais para a necessidade de $\mathrm{CP}{ }^{(8)}$ Corrobora estes dados um estudo populacional na Espanha que também encontrou maior prevalência de quadros de fragilidade e demenciais no contexto da atenção primária. ${ }^{(9)}$

Esses dados são compatíveis com o envelhecimento da população brasileira, que cursa com aumento de doenças crônicas não transmissíveis. Associa-se a isso o fato de que a prevalência das demências dobra a cada cinco anos após os 60 anos de idade. ${ }^{(10)}$

No presente estudo a prevalência de acidente vascular encefálico / demência vascular foi maior do que a prevalência de demência de Alzheimer; no entanto, a causa mais comum de demência é a DA (60 a 70\% dos casos). ${ }^{(11)}$ Uma explicação possível para esse fenômeno é de que a DV apresenta uma epidemiologia de difícil caracterização devido aos seguintes motivos: grande variação nas características das populações estudadas, falta de critérios diagnósticos universais, dificuldade para definir DV e a existência de quadros mistos (demência mista). ${ }^{(12)}$ Estudos neuropatológicos post mortem mostram que frequentemente ocorrem lesões da DA associadas à DV (casos de demência mista não diagnosticadas em vida), ${ }^{(12)}$ não se podendo excluir que os casos diagnosticados neste estudo como demência vascular exclusiva não apresentassem um componente de DA associado.

A prevalência de SI no sexo masculino foi de $6,1 \%$, enquanto no feminino foi de $13,1 \%$, com significância estatística $p=0,0218$. Corroboram estes dados, os levantados pela Pesquisa Nacional por Amostras de Domicílios (PNAD) que mostra que a prevalência de incapacidade funcional é mais elevada nas mulheres, o que evidencia que, apesar de viverem mais, suportam um maior número de problemas de saúde. ${ }^{(13)}$

Quanto aos critérios diagnósticos para SI, $100 \%$ dos pacientes cursaram com dupla incontinência, $83,9 \%$ disfagia, $73,2 \%$ afasia e $55,4 \%$ lesões por pressão. Esses dados se assemelham ao 
estudo de prevalência de SI em uma enfermaria de geriatria que mostrou maior prevalência de dupla incontinência em $100 \%$, seguida por afasia (75\%) e lesões por pressão $(66,6 \%) .{ }^{(3)}$

No estudo, $83,9 \%$ apresentavam disfagia leve a grave, no entanto apenas $26,79 \%$ estavam com via alternativa de alimentação. A decisão da inserção de vias alternativas de alimentação constitui uma difícil decisão aos profissionais de saúde e aos familiares. Conforme a American Geriatrics Society, em populações vulneráveis como os idosos demenciados em estágio avançado, que se encaixam no perfil dos pacientes com SI, os tubos de alimentação não são recomendados, pois sua inserção se associa a reincidências de lesão por pressão e ao aumento dos cuidados em saúde. ${ }^{(14)}$ A nutrição e a hidratação artificial têm sido consideradas uma demonstração de cuidado e carinho e renunciar tais medidas tem sido interpretado como negligência e abandono. ${ }^{(15)}$

Dos critérios menores, a dupla incontinência teve uma prevalência de $100 \%$ nos pacientes com SI, sendo a incontinência o ponto de partida de um estudo conduzido na cidade de São Paulo, que identificou indivíduos com indicação de $\mathrm{CP}$ a partir de um programa de dispensação de insumos para incontinência urinária e fecal. A amostra foi de 160 indivíduos, tendo um predomínio de casos neurológicos crônicos e neurodegenerativos, como doença cerebrovascular, doença de Alzheimer, doença de Parkinson e outras demências. ${ }^{(16)}$

Entre os pacientes com SI, 55,36\% não estavam em cuidados paliativos, dos quais $8,93 \%$ por não aceitação da família. Partindo do princípio de que todos os pacientes com SI apresentam indicação clínica de cuidados paliativos, esse número mostra que o total de pacientes que receberam o tratamento adequado estava muito aquém do ideal. Esse dado é corroborado pela estimativa da ONU de que somente $14 \%$ das pessoas que precisam de cuidados paliativos os recebam efetivamente ${ }^{(17)}$ e na publicação de 2015 do jornal The Economist que mostrou a informação de que o Brasil está em $42^{\circ}$ lugar em um ranking que mostra a qualidade de morte entre os países. ${ }^{(18)}$
Ao correlacionar as causas de imobilismo e inclusão em cuidados paliativos, observou-se que apenas a demência mista teve um $p$ significativo: $p=0,0344$; todas as outras condições, como acidente vascular encefálico, demência vascular, demência de Alzheimer, trauma de crânio e parkinsonismo não tiveram associação, ou seja, ter SI com estas causas não assegura sua inclusão em CP. Apesar de a doença cerebrovascular ser a principal causa de incapacidade e morte, as abordagens de CP ainda são limitadas, e mesmo quando incluídas, são realizadas nos estágios finais da doença. ${ }^{(19)}$

No trauma de crânio, como causa de SI, $83 \%$ não estavam em cuidados paliativos (comparado a $52 \%$ dentre os que não apresentaram trauma de crânio). Isso pode ser explicado pelo fato de que entre os pacientes com trauma de crânio 83,3\% eram jovens com idade entre 21 e 51 anos, o que pode tornar mais difícil a aceitação por parte da família e da própria equipe médica em relação à implantação de CP.

A abordagem no domicilio em CP pressupõe o cuidado integral ao indivíduo e amplia a atenção para o contexto pessoal e familiar na busca de influenciar positivamente na condição de saúde e qualidade de vida, oferecendo orientação, tratamento e cuidado. ${ }^{(20)}$ Apesar da grande potencialidade de prestar um cuidado holístico, o profissional se distancia por ter dificuldades em estimar sobrevivência, tendência dos médicos em superestimar o tempo de sobrevida dos pacientes com doenças avançadas e falta de capacitação em medicina paliativa. ${ }^{(21)}$ No presente estudo, ressalta-se outra causa que é a falta de conhecimento da SI e a indicação de tratamento paliativo para essa entidade.

A principal limitação do estudo consistiu na escassez de literatura a respeito da SI e da metodologia adotada que foi documental.

\section{Conclusão}

A SI foi associada ao sexo feminino, a média de idade foi 72,43 anos e a prevalência foi de $9,9 \%$, dos quais somente $44,46 \%$ estavam em cuidados paliativos. A frequência dos critérios diagnósticos 
menores foram $100 \%$ dupla incontinência, $83,9 \%$ disfagia, $73,2 \%$ afasia e $55,4 \%$ lesões por pressão.

A principal causa da SI foi AVE/demência vascular (41\%), seguido da demência de Alzheimer (23\%), trauma de crânio (11\%) e demência mista (7\%). O diagnóstico demência mista foi a única causa de imobilismo associada com indicação cuidados paliativos.

Ações devem ser desenvolvidas para que os profissionais que trabalhem com atendimento domiciliar possam reconhecer a SI, bem como a indicação de $\mathrm{CP}$, a fim de ofertar o melhor atendimento possível, desenvolvendo protocolos institucionais de cuidados.

\section{Referências}

1 Leduc MMS, Leduc VR, Suguino MM. Imobilidade e síndrome da imobilização. In: Freitas EV, Py L. Tratado de Geriatria. 4a ed. São Paulo: Editora Guanabara; 2016. p. 10681069.

2 Biolo G, CiocchiB, Stulle M, Piccoli A, Lorenzon $\mathrm{S}$, Dal Mas V, et al. Metabolic consequences of physical inactivity. J Ren Nutr. 2015;15(1):4953. doi: 10.1053/j.jm.2004.09.009.

3 Ribeiro CA, Silva DAM, Rizzo LA, Ventura MM. Frequência da síndrome de imobilidade em uma enfermaria de geriatria. Geriatr. Gerontol. [Internet]. 2011 [citado em 2019 jan 17]; 5(3):136-9. Disponível em: https://sbgg. org.br//wp-content/uploads/2014/10/2011-3.pdf

4 Moro JV, Caliri MHL. Úlcera por pressão após a alta hospitalar e o cuidado em domicílio. Esc. Anna Nery. 2016;20(3):1-6. doi:10.5935/14148145.20160058 .

5 Ministério da Saúde (BR). Portaria de Consolidação $\mathrm{n}^{\circ} 5$, de 28 de setembro de 2017. Consolidação das normas sobre as ações e os serviços de saúde do Sistema Único de Saúde [Internet]; 2017. [citado 2019 jan 19]. Disponível em: https://bvsms. saude.gov.br/bvs/saudelegis/gm/2017/ prc0005_03_10_2017.html
6 Namoah AM. Palliative care definition. Houston: International Association For Hospice \& Palliative Care; 2019.

7 Marcucci FCI, Martins VM, Barros EML, Perilla AB, Brun MM, Cabrera MAS. Functional capacity of patients indicated for palliative care in primary care. Geriatr Gerontol Aging. 2018;12(3):159-65. doi:10.5327/ z2447-211520181800026.

8 Lucchetti G, Badan AM Neto, Ramos SAC, Faria LFC, Granero AL, Pires SL, et al. Uso de uma escala de triagem para cuidados paliativos nos idosos de uma instituição de longa permanência. Geriatr Gerontol Aging [Internet]. 2009 [citado 20195 jun]; 3(3): 1048. Disponível em: www.ggaging.com/exportpdf/299/v3n3a02.pdf

9 Gómez-Batiste X, Martínez-Muñoz M, Blay $\mathrm{C}$, Amblàs J, Vila L, Costa X, et al. Prevalence and characteristics of patients with advanced chronic conditions in need of palliative care in the general population: a cross-sectional study. Palliat Med. 2014;28(4):302-11. doi: $10.1177 / 0269216313518266$.

10 Kaycee MS, Yaffe K. Diagnóstico e tratamento, comprometimento cognitivo e demência. 2a ed. Porto Alegre: Editora AMGH; 2015. p. 123.

11 Holtzman DM, Morris JC, Goate AM. Alzheimer's Disease: the challenge of the second century. Sci Transl Med. 2011;3(3):217. doi:10.1126/scitranslmed.3002369.

12 Engelhardt E, Tocquer C, Andre C, Moreira DM, Okamoto IH, Cavalcanti JS. Demência Vascular. Critérios diagnósticos e exames complementares. Demente Neuropsychol [Internet]. 2011 June [citado 2019 ju 5];5(1):49-77. Disponível em: https://www. redalyc.org/html/3395/339529025006/

13 Parahyba MI, Veras R. Diferenciais sociodemográficos no declínio funcional em mobilidade física entre os idosos no Brasil. Ciênc. Saúde Coletiva [Internet]. 2008 [citado 2019 jun. 5];13(4):1257-64. Disponível em: http://www.scielo.br/scielo.php?pid=S1413$81232008000400022 \& \mathrm{script}=\mathrm{sci}$ abstract\&tlng $=\mathrm{pt}$ 
14 American Geriatrics Society Ethics Committee, Clinical Practice and Models of Care Committee. American Geriatrics Society feeding tubes in advanced dementia position statement. J Am Geriatr Soc. 2014;62(8):159093. doi: http://dx.doi.org/10.1111/jgs.12924

$15 \mathrm{Li}$ I. Feeding tubes in patients with severe dementia. Am Fam Physician. 2002;65(8):1605-10.

16 Paz CRP. Cuidados Paliativos na atenção primária à saúde: novos desafios. São Paulo: Universidade de São Paulo; 2013.

17 Atty ATM, Tomazelli JG. Cuidados paliativos na atenção domiciliar em pacientes oncológicos no Brasil. Rev Saúde Debate [Internet]. 2018 [citado 2019 jun 5];42(116):225-36. Disponível em: https:/www.scielosp.org/ article/ssm/content/raw/?resource_ssm path=/media/assets/sdeb/v42n116/0103-1104sdeb-42-116-0225.pdf

18 Vasconcelos GB, Pereira PM. Cuidados paliativos na atenção domiciliar: uma revisão bibliográfica. Rev.Adm. Saúde. 2018;18(70):118. doi:http://dx.doi.org/10.23973/ras. 70.85

19 Creutzfeldt CJ, Holloway RG, Walker M. Symptomatic and palliative care for stroke survivors. J Gen Intern Med. 2012;27(7):85360. doi: 10.1007/s11606-011-1966-4.

20 Valente SH, Teixeira MB. Estudo fenomenológico sobre a visita domiciliária do enfermeiro à família no processo de terminalidade. Rev Esc Enferm USP [Internet]. 2009 [citado 2019 jun 5];43(3):65561. Disponível em: http://www.scielo.br/pdf/ reeusp/v43n3/a22v43n3.pdf

21 Ferraz-Falcao JR, Cadaval-Rodríguez T, Rodríguez-Iglesia RM, Campo-Pérez VM. Necesidad de cuidados paliativos en la población inmovilizada de un centro de salud de vigo. Seemergen. 2014;6(17):1-10. doi: 10.1016/j.semerg.2014.06.017.

Recebido em: 13 jun. 2019

Aceito em: 28 nov. 2019 
Espindola, R.; Cechinel, C.; Moretti, R. P. 